Pacific

Journal of

Mathematics

FREDHOLM PROPERTIES OF TOEPLITZ OPERATORS ON DIRICHLET SPACES

Guangfu CaO 


\title{
FREDHOLM PROPERTIES OF TOEPLITZ OPERATORS ON DIRICHLET SPACES
}

\author{
Guangfu CaO
}

\begin{abstract}
In this paper, the Fredholm properties of some Toeplitz operators on Dirichlet spaces be discussed, and the essential spectra of Toeplitz operators with symbols in $C^{1}$ or $H_{1}^{\infty}+C^{1}$ be computed.
\end{abstract}

\section{Introduction.}

Let $\mathbb{D}$ be the unit disk in the complex plane $\mathbb{C}, d A=\frac{1}{\pi} d x d y$ be the normalized area measure on $\mathbb{D}$. $L^{2,1}$ is the space of functions $u: \mathbb{D} \rightarrow \mathbb{C}$, for which the norm

$$
\|u\|_{\frac{1}{2}}=\left[\int_{\mathbb{D}}\left(\left|\frac{\partial u}{\partial z}(z)\right|^{2}+\left|\frac{\partial u}{\partial \bar{z}}(z)\right|^{2}\right) d A\right]^{\frac{1}{2}}<\infty .
$$

$L^{2,1}$ is a Hilbert space with the inner product

$$
\langle u, v\rangle_{\frac{1}{2}}=\left\langle\frac{\partial u}{\partial z}, \frac{\partial v}{\partial z}\right\rangle_{L^{2}(d A)}+\left\langle\frac{\partial u}{\partial \bar{z}}, \frac{\partial v}{\partial \bar{z}}\right\rangle_{L^{2}(d A)} .
$$

The Dirichlet space, $\mathcal{D}$, is the subspace of all analytic functions $g$ in $L^{2,1}$ with $g(0)=0$. Let $P$ be the orthogonal projection from $L^{2,1}$ into $\mathcal{D}$. $P$ is an integral operator represented by

$$
P(u)(w)=\int_{\mathbb{D}} \frac{\partial u}{\partial z} \frac{\overline{\partial K(z, w)}}{\partial z} d A
$$

where $K(z, w)=\sum_{k=1}^{\infty} \frac{z^{k} \bar{w}^{k}}{k}$ is the reproducing kernel of $\mathcal{D}$ (see R. Rochberg and Z.J. Wu [5] and $\mathrm{Wu}[7])$. Let $G$ be a domain in $\mathbb{C}$, define

$$
\begin{aligned}
& C^{1}(G)=\left\{u \mid u, \frac{\partial u}{\partial z}, \frac{\partial u}{\partial \bar{z}} \in C(G)\right\}, \\
& C^{1}(\bar{G})=\left\{u \mid u, \frac{\partial u}{\partial z}, \frac{\partial u}{\partial \bar{z}} \in C(\bar{G})\right\},
\end{aligned}
$$

and

$$
H_{1}^{\infty}(G)=\left\{f \in H(G) \mid f^{\prime} \in H^{\infty}(G)\right\}
$$


where $C(G)$ (or $C(\bar{G}))$ and $H(G)$ are respectively the set of continuous functions on $G$ (or $\bar{G})$ and the one of analytic functions on $G$.

In [5] and [7], R. Rochberg and Z.J. Wu define the Toeplitz operator with symbol $\mu$ as

$$
T_{\mu} f=\int_{\mathbb{D}} f^{\prime}(z) \frac{\overline{\partial K(z, w)}}{\partial z} d \mu \quad(\forall f \in \mathcal{D}),
$$

where $\mu$ is a measure on $\mathbb{D}$. If $d \mu=\varphi d A, \varphi \in L^{\infty}(\mathbb{D}, d A)$, one can define

$$
T_{\varphi} f=\int_{\mathbb{D}} f^{\prime}(z) \frac{\overline{\partial K(z, w)}}{\partial z} \varphi d A
$$

We see easily that $T_{\varphi} f \neq P(\varphi f)$ in general, in fact, $P(\varphi f)$ may be undefined for $\varphi \in L^{\infty}(\mathbb{D}, d A)$. However, it seems to be more natural that the Toeplitz operators be defined as the form $P(\varphi f)$. In this paper, we try to define the Toeplitz operators with some special symbols as following

Definition 1. Suppose $\varphi \in C^{1}(\mathbb{D})$, the operator

$$
T_{\varphi} f(w)=P(\varphi f)(w)=\langle\varphi f, K\rangle_{\frac{1}{2}}=\int_{\mathbb{D}} \frac{\partial(\varphi f)}{\partial z} \frac{\overline{\partial K(z, w)}}{\partial z} d A(z)
$$

is said to be the Toeplitz operator with symbol $\varphi$.

We will compute the spectra and essential spectra of these operators. For convenience, we use frequently the notation $\tilde{T}_{\varphi}$ to denote the Toeplitz operator with symbol $\varphi$ on Bergman space.

By the way, our results are also true for Toeplitz operators on weighted Dirichlet spaces $\mathcal{D}_{\alpha}(\alpha<1)$. If $\alpha=\frac{1}{2}$, then $\mathcal{D}_{\frac{1}{2}}=\mathcal{D}$, the usual Dirichlet space (c.f. [5], [7]).

\section{Toeplitz operators with symbols in $C^{1}(\overline{\mathbb{D}})$.}

Throughout this paper, we use the symbol " $\langle.,$.$\rangle " to represent the inner$ product in $L^{2}(\mathbb{D}, d A)$, and " $\langle., .\rangle_{\frac{1}{2}}$ " to that in $\mathcal{D}$.

Define the norm in $C^{1}(\overline{\mathbb{D}})$ as

$$
\|\varphi\|_{*}=\max _{z \in \overline{\mathbb{D}}} \max \left\{|\varphi|,\left|\frac{\partial \varphi}{\partial z}\right|,\left|\frac{\partial \varphi}{\partial \bar{z}}\right|\right\}, \quad\left(\forall \varphi \in C^{1}(\overline{\mathbb{D}})\right) .
$$

It is well-known that $C^{1}(\overline{\mathbb{D}})$ is a norm-closed algebra relative to $\|\cdot\|_{*}$.

Lemma 1. For any $\varphi \in C^{1}(\overline{\mathbb{D}}), H_{\varphi} f=:(I-P)(\varphi f)(\forall f \in \mathcal{D})$ is a compact operator from $\mathcal{D}$ to $\mathcal{D}^{\perp}$. 
Proof. For any $f \in \mathcal{D}, g \in \mathcal{D}^{\perp}$

$$
\begin{aligned}
\left\langle H_{\varphi} f, g\right\rangle_{\frac{1}{2}}=\langle\varphi f, g\rangle_{\frac{1}{2}} & =\left\langle\frac{\partial \varphi}{\partial z} f+\varphi \frac{\partial f}{\partial z}, \frac{\partial g}{\partial z}\right\rangle+\left\langle\frac{\partial \varphi}{\partial \bar{z}} f, \frac{\partial g}{\partial \bar{z}}\right\rangle \\
& =\left\langle\frac{\partial \varphi}{\partial z} f, \frac{\partial g}{\partial z}\right\rangle+\left\langle\varphi \frac{\partial f}{\partial z}, \frac{\partial g}{\partial z}\right\rangle+\left\langle\frac{\partial \varphi}{\partial \bar{z}} f, \frac{\partial g}{\partial \bar{z}}\right\rangle .
\end{aligned}
$$

Note $g \perp \mathcal{D}$, so $\frac{\partial g}{\partial z} \perp L_{a}^{2}\left(L_{a}^{2}\right.$ is the classical Bergman space on $\left.\mathbb{D}\right)$. Let $P_{a}$ is the orthogonal projection from $L^{2}(\mathbb{D}, d A)$ to $L_{a}^{2}$, then for any $f \in \mathcal{D}$ and $g \in \mathcal{D}^{\perp}$, we have

$$
\left\langle\varphi f^{\prime}, \frac{\partial g}{\partial z}\right\rangle=\left\langle\left(I-P_{a}\right)\left(\varphi f^{\prime}\right), \frac{\partial g}{\partial z}\right\rangle=\left\langle\tilde{H}_{\varphi} f^{\prime}, \frac{\partial g}{\partial z}\right\rangle,
$$

where $\widetilde{H}_{\varphi}$ is the Hankel operator from $L_{a}^{2}$ to $L_{a}^{2 \perp}$ with symbol $\varphi$. Since $\varphi \in C^{1}(\overline{\mathbb{D}}), \tilde{H}_{\varphi}$ is a compact operator. Now assume that $\left\{f_{k}\right\} \subset \mathcal{D}$ is a sequence which converges weakly 0 and satisfies $\left\|f_{k}\right\|_{\mathcal{D}}=1$, we prove first that $\left\|f_{k}\right\|_{L^{2}} \rightarrow 0(k \rightarrow \infty)$. In fact, without loss of generality, assume $f_{k}(z)=$ $\sum_{n=1}^{\infty} a_{n}^{(k)} z^{k}$, then $a_{n}^{(k)} \rightarrow 0(k \rightarrow \infty, \forall n)$ by $f_{k} \stackrel{w}{\rightarrow} 0$. Note $\left\|f_{k}\right\|_{\mathcal{D}}^{2}=\left\|f_{k}^{\prime}\right\|_{L^{2}}^{2}=$ 1 , so $\sum_{n=1}^{\infty}\left|a_{n}^{(k)}\right|^{2} n=1(\forall k)$, thus $\sum_{n=1}^{\infty}\left|a_{n}^{(k)}\right|^{2} \leq 1$, furthermore, for any $\epsilon>$ 0 , there is a $N_{0}$ which is independent on $k$ such that $\sum_{n \geq N_{0}+1}^{\infty}\left|a_{n}^{(k)}\right|^{2} \frac{1}{n+1}<\frac{\epsilon}{2}$. Fix such a $N_{0}$, then

$$
\left\|f_{k}\right\|_{L^{2}}^{2}=\sum_{n=1}^{\infty}\left|a_{n}^{(k)}\right|^{2} \frac{1}{n+1} \leq \sum_{n=1}^{N_{0}}\left|a_{n}^{(k)}\right|^{2} \frac{1}{n+1}+\frac{\epsilon}{2} .
$$

Since $a_{n}^{(k)} \rightarrow 0(\forall n, k \rightarrow \infty)$, there is a $K_{0}$ such that for any $k>K_{0}$, $\sum_{n=1}^{N_{0}}\left|a_{n}^{(k)}\right|^{2} \frac{1}{n+1}<\frac{\epsilon}{2}$, thus $\left\|f_{k}\right\|_{L^{2}}^{2}<\frac{\epsilon}{2}\left(k>K_{0}\right)$, hence $\left\|f_{k}\right\|_{L^{2}} \rightarrow 0$. Note

$$
\begin{aligned}
\left\|H_{\varphi} f_{k}\right\|_{L^{2,1}}^{2}= & \left\langle H_{\varphi} f_{k}, H_{\varphi} f_{k}\right\rangle_{\frac{1}{2}} \\
\leq & \left\|\frac{\partial \varphi}{\partial z} f_{k}\right\|_{L^{2}}\left\|H_{\varphi} f_{k}\right\|_{L^{2,1}}+\left\|\tilde{H}_{\varphi} f_{k}^{\prime}\right\|_{L^{2}}\left\|H_{\varphi} f_{k}\right\|_{L^{2,1}} \\
& +\left\|\frac{\partial \varphi}{\partial \bar{z}} f_{k}\right\|_{L^{2}}\left\|H_{\varphi} f_{k}\right\|_{L^{2,1}} \\
\leq & \|\varphi\|_{*}\left\|f_{k}\right\|_{L^{2}}\left\|H_{\varphi} f_{k}\right\|_{L^{2,1}}+\left\|\tilde{H}_{\varphi} f_{k}^{\prime}\right\|_{L^{2}}\left\|H_{\varphi} f_{k}\right\|_{L^{2,1}} \\
& +\|\varphi\|_{*}\left\|f_{k}\right\|_{L^{2}}\left\|H_{\varphi} f_{k}\right\|_{L^{2,1}},
\end{aligned}
$$

we know easily that $\left\|H_{\varphi} f_{k}\right\|_{L^{2,1}} \rightarrow 0$. This shows that $H_{\varphi}$ is compact.

Proposition 2. For any $\varphi \in C^{1}(\overline{\mathbb{D}}), T_{\varphi}$ is a bounded operator on $\mathcal{D}$. 
Proof. For any $f, g \in \mathcal{D}$,

$$
\left\langle T_{\varphi} f, g\right\rangle_{\frac{1}{2}}=\langle\varphi f, g\rangle_{\frac{1}{2}}=\left\langle\frac{\partial(\varphi f)}{\partial z}, g^{\prime}\right\rangle=\left\langle\frac{\partial \varphi}{\partial z} f, g^{\prime}\right\rangle+\left\langle\varphi f^{\prime}, g^{\prime}\right\rangle,
$$

hence

$$
\begin{aligned}
\left|\left\langle T_{\varphi} f, g\right\rangle_{\frac{1}{2}}\right| & \leq\left\|\frac{\partial \varphi}{\partial z} f\right\|_{L^{2}}\left\|g^{\prime}\right\|_{L^{2}}+\left\|\varphi f^{\prime}\right\|_{L^{2}}\left\|g^{\prime}\right\|_{L^{2}} \\
& \leq\|\varphi\|_{*}\left(\|f\|_{L^{2}}\|g\|_{\mathcal{D}}+\|f\|_{\mathcal{D}}\|g\|_{\mathcal{D}}\right) .
\end{aligned}
$$

It is not difficult to check $\|f\|_{L^{2}} \leq\|f\|_{\mathcal{D}}$ for any $f \in \mathcal{D}$ (since $f(0)=0$ for any $f \in \mathcal{D})$, hence $\left|\left\langle T_{\varphi} f, g\right\rangle_{\frac{1}{2}}\right| \leq 2\|\varphi\|_{*}\|f\|_{\mathcal{D}}\|g\|_{\mathcal{D}}$. This shows that $\left\|T_{\varphi}\right\| \leq$ $2\|\varphi\|_{*}$.

Remark. In general, $\left\|T_{\varphi}\right\| \neq\|\varphi\|_{*}$ even $\varphi \in C^{1}(\mathbb{D}) \cap A(\mathbb{D})(A(\mathbb{D})$ be the disk algebra), it may be that $\|\varphi\|_{C^{1}}<\left\|T_{\varphi}\right\|<2\|\varphi\|_{C^{1}}$. For instance, if $\varphi(z)=z$, then $\|\varphi\|_{*}=\|\varphi\|_{\infty}=1$, but $\left\|T_{\varphi}\right\| \geq \sqrt{2}$.

Proposition 3. Let $L^{\infty, 1}=\left\{f \in L^{2,1} \mid f, \frac{\partial f}{\partial z}, \frac{\partial f}{\partial \bar{z}} \in L^{\infty}(\mathbb{D}, d A)\right\}$, then for any $\varphi \in L^{\infty, 1}$ and $\psi \in C^{1}(\overline{\mathbb{D}}) T_{\varphi} T_{\psi}-T_{\varphi \psi} \in \mathcal{K}(\mathcal{D})$ (the compact operator algebra on $\mathcal{D}$.)

Proof. For any $f, g \in \mathcal{D}$, as a direct computing, we see that

$$
\begin{aligned}
\left\langle\left(T_{\varphi} T_{\psi}-T_{\varphi \psi}\right) f, g\right\rangle_{\frac{1}{2}}= & \left\langle\frac{\partial \varphi}{\partial z} T_{\psi} f, \frac{\partial g}{\partial z}\right\rangle-\left\langle\frac{\partial \varphi}{\partial z}(\psi f), \frac{\partial g}{\partial z}\right\rangle \\
& +\left\langle\varphi \frac{\partial\left(-H_{\psi} f\right)}{\partial z}, \frac{\partial g}{\partial z}\right\rangle,
\end{aligned}
$$

set $g=\left(T_{\varphi} T_{\psi}-T_{\varphi \psi}\right) f$, we have

$$
\begin{aligned}
\left\|\left(T_{\varphi} T_{\psi}-T_{\varphi \psi}\right) f\right\|_{\mathcal{D}}^{2} \leq & \|\varphi\|_{*}\left[\left\|T_{\psi} f\right\|_{L^{2}}+\|\psi\|_{*}\|f\|_{L^{2}}\right. \\
& \left.+\left\|H_{\psi} f\right\|_{L^{2,1}}\right]\left\|\left(T_{\varphi} T_{\psi}-T_{\varphi \psi}\right) f\right\|_{\mathcal{D}}
\end{aligned}
$$

Note for any sequence $\left\{f_{k}\right\} \subset \mathcal{D}$ which converges weakly 0 and satisfies $\left\|f_{k}\right\|_{\mathcal{D}}=1$ (certainly, $T_{\psi} f_{k} \stackrel{w}{\rightarrow} 0$ in $\mathcal{D}$ ), $\left\|T_{\psi} f_{k}\right\|_{L^{2}} \rightarrow 0,\left\|f_{k}\right\|_{L^{2}} \rightarrow 0$, hence

$$
\left\|\left(T_{\varphi} T_{\psi}-T_{\varphi \psi}\right) f_{k}\right\|_{\mathcal{D}} \rightarrow 0
$$

by $H_{\psi}$ is compact. Futhermore, $T_{\varphi} T_{\psi}-T_{\varphi \psi}$ is compact.

Lemma 4. For any $\varphi \in L^{\infty, 1}, T_{\varphi}^{*}-T_{\bar{\varphi}} \in \mathcal{K}(\mathcal{D})$.

Proof. Similar to the proof of Proposition 2, it is easy to see that $T_{\varphi}$ is bounded for any $\varphi \in L^{\infty, 1}$. Now assume $\varphi \in L^{\infty, 1}$, then for any $f \cdot g \in \mathcal{D}$, 
$\left\langle\left(T_{\varphi}^{*}-T_{\bar{\varphi}}\right) f \cdot g\right\rangle_{\frac{1}{2}}=\left\langle\frac{\partial f}{\partial z}, \frac{\partial \varphi}{\partial z} g\right\rangle-\left\langle f, \frac{\partial \varphi}{\partial \bar{z}} \frac{\partial g}{\partial z}\right\rangle$. Thus

$$
\begin{aligned}
\left|\left\langle\left(T_{\varphi}^{*}-T_{\bar{\varphi}}\right) f, g\right\rangle_{\frac{1}{2}}\right| & \leq\left\|\frac{\partial f}{\partial z}\right\|_{L^{2}}\left\|\frac{\partial \varphi}{\partial z} g\right\|_{L^{2}}+\|f\|_{L^{2}}\left\|\frac{\partial \varphi}{\partial \bar{z}} \frac{\partial g}{\partial z}\right\|_{L^{2}} \\
& \leq\|\varphi\|_{*}\|g\|_{L^{2}}\|f\|_{\mathcal{D}}+\|\varphi\|_{*}\|f\|_{L^{2}}\|g\|_{\mathcal{D}},
\end{aligned}
$$

where $\|\varphi\|_{*}=\operatorname{esssup}_{z \in \mathbb{D}} \max \left\{|\varphi(z)|,\left|\frac{\partial \varphi}{\partial z}(z)\right|,\left|\frac{\partial \varphi}{\partial \bar{z}}(z)\right|\right\}$. Hence for any $\left\{f_{k}\right\} \subset$ $\mathcal{D},\left\|f_{k}\right\|=1, f_{k} \stackrel{w}{\rightarrow} 0$, we have

$\left\|\left(T_{\varphi}^{*}-T_{\bar{\varphi}}\right) f_{k}\right\|_{\mathcal{D}}^{2} \leq\|\varphi\|_{*}\left\|\left(T_{\varphi}^{*}-T_{\bar{\varphi}}\right) f_{k}\right\|_{L^{2}}\left\|f_{k}\right\|_{\mathcal{D}}+\|\varphi\|_{*}\left\|f_{k}\right\|_{L^{2}}\left\|\left(T_{\varphi}^{*}-T_{\bar{\varphi}}\right) f_{k}\right\|_{\mathcal{D}}$.

Note $\left(T_{\varphi}^{*}-T_{\bar{\varphi}}\right) f_{k} \stackrel{w}{\rightarrow} 0$, so $\left\|\left(T_{\varphi}^{*}-T_{\bar{\varphi}}\right) f_{k}\right\|_{L^{2}} \rightarrow 0$. Consequently, $\|\left(T_{\varphi}^{*}-\right.$ $\left.T_{\bar{\varphi}}\right) f_{k} \|_{\mathcal{D}} \rightarrow 0$. This shows that $T_{\varphi}^{*}-T_{\bar{\varphi}}$ is compact.

Remark. It is well-known that $T_{\varphi}^{*}=T_{\bar{\varphi}}$ for any $\varphi \in L^{\infty}(\mathbb{D}, d A)$ on $L_{a}^{2}$, but it is not difficult to check $T_{\varphi}^{*} \neq T_{\bar{\varphi}}$ in general. For example, if $\varphi(z)=\sum_{n=1}^{\infty} \frac{z^{n}}{n^{2}}$, then $\varphi \in H^{\infty}$, but $\left\|\varphi^{\prime}\right\|_{\infty}=\infty$, one can check easily that $T_{\varphi}$ is unbounded, however, $T_{\bar{\varphi}}$ is always bounded for every $\varphi \in H^{\infty}$, so is $T_{\bar{\varphi}}^{*}$, hence $T_{\bar{\varphi}}^{*}-T_{\varphi}$ is unbounded.

Proposition 5. If $\varphi \in C^{1}(\overline{\mathbb{D}})$, then $T_{\varphi}$ is a compact operator on $\mathcal{D}$ if and only if $\left.\varphi\right|_{\partial \mathbb{D}} \equiv 0$.

Proof. Assume $\left.\varphi\right|_{\partial \mathbb{D}} \equiv 0$, then $\widetilde{T_{|\varphi|^{2}}}$ is a compact operator on $L_{a}^{2}(\mathbb{D}, d A)$, so for any $\left\{f_{k}\right\} \subset L_{a}^{2}, f_{k} \stackrel{w}{\rightarrow} 0,\left\|f_{k}\right\|_{L^{2}}=1$, we have $\left\|\tilde{T_{|\varphi|}} f_{k}\right\|_{L^{2}} \rightarrow 0(k \rightarrow \infty)$. If $T_{\varphi}$ is not compact on $\mathcal{D}$, then there is a sequence $\left\{F_{k}\right\} \subset \mathcal{D},\left\|F_{k}\right\|_{\mathcal{D}}=1$, $F_{k} \stackrel{w}{\rightarrow} 0$ such that $\left\|T_{\varphi} F_{k}\right\|_{\mathcal{D}} \nrightarrow 0$, thus $\left\|\varphi F_{k}\right\|_{L^{2,1}} \nrightarrow 0$, furthermore

$$
\left\langle\frac{\partial\left(\varphi F_{k}\right)}{\partial z}, \frac{\partial\left(\varphi F_{k}\right)}{\partial z}\right\rangle+\left\langle\frac{\partial\left(\varphi F_{k}\right)}{\partial \bar{z}}, \frac{\partial\left(\varphi F_{k}\right)}{\partial \bar{z}}\right\rangle \nrightarrow 0 .
$$

Note

thus

$$
\frac{\partial\left(\varphi F_{k}\right)}{\partial z}=\frac{\partial \varphi}{\partial z} F_{k}+\varphi \frac{\partial F_{k}}{\partial z}, \frac{\partial\left(\varphi F_{k}\right)}{\partial \bar{z}}=\frac{\partial \varphi}{\partial \bar{z}} F_{k},
$$

$$
\left\langle\frac{\partial\left(\varphi F_{k}\right)}{\partial \bar{z}}, \frac{\partial\left(\varphi F_{k}\right)}{\partial \bar{z}}\right\rangle \leq\|\varphi\|_{*}^{2}\left\|F_{k}\right\|_{L^{2}}^{2} \rightarrow 0,
$$

and

$$
\begin{aligned}
\left\|\frac{\partial\left(\varphi F_{k}\right)}{\partial z}\right\|_{L^{2}}^{2}= & \left\langle\frac{\partial \varphi}{\partial z} F_{k}, \frac{\partial \varphi}{\partial z} F_{k}\right\rangle+\left\langle\frac{\partial \varphi}{\partial z} F_{k}, \varphi F_{k}^{\prime}\right\rangle \\
& +\left\langle\varphi F_{k}^{\prime}, \frac{\partial \varphi}{\partial z} F_{k}\right\rangle+\left\langle\varphi F_{k}^{\prime}, \varphi F_{k}^{\prime}\right\rangle .
\end{aligned}
$$

Since $\left\|F_{k}^{\prime}\right\|_{L^{2}}=\left\|F_{k}\right\|_{\mathcal{D}}=1$, and $F_{k}^{\prime} \stackrel{w}{\rightarrow} 0$ in $L_{a}^{2}$,

$$
\left\langle\varphi F_{k}^{\prime}, \varphi F_{k}^{\prime}\right\rangle=\left\langle|\varphi|^{2} F_{k}^{\prime}, F_{k}^{\prime}\right\rangle=\left\langle T_{|\varphi|^{2}} F_{k}^{\prime}, F_{k}^{\prime}\right\rangle \rightarrow 0,
$$


consequently, $\left\|\frac{\partial\left(\varphi F_{k}\right)}{\partial z}\right\|_{L^{2}}^{2} \rightarrow 0$ by $\left|\left\langle\frac{\partial \varphi}{\partial z} F_{k}, \frac{\partial \varphi}{\partial z} F_{k}\right\rangle\right| \leq\|\varphi\|_{*}^{2}\left\|F_{k}\right\|_{L^{2}}^{2}$ and $\left|\left\langle\frac{\partial \varphi}{\partial z} F_{k}, \varphi F_{k}^{\prime}\right\rangle\right| \leq\|\varphi\|_{*}^{2}\left\|F_{k}\right\|_{L^{2}}\left\|F_{k}^{\prime}\right\|_{L^{2}}$, and $\left\|F_{k}\right\|_{L^{2}} \rightarrow 0$. This contradicts that $\left\|\varphi F_{k}\right\|_{L^{2,1}} \nrightarrow 0$. Hence $T_{\varphi}$ must be a compact operator on $\mathcal{D}$.

Conversely, assume that $T_{\varphi}$ is compact on $\mathcal{D}$. We need to prove that $\left.\varphi\right|_{\partial \mathbb{D}}=0$. Otherwise, $\widetilde{T}_{|\varphi|}$ is not compact on $L_{a}^{2}(\mathbb{D})$, thus there is a sequence $\left\{f_{k}\right\} \subset L_{a}^{2},\left\|f_{k}\right\|_{L^{2}}=1, f_{k} \stackrel{w}{\rightarrow} 0$ such that $\left\|\tilde{T_{|\varphi|}} f_{k}\right\|_{L^{2}} \nrightarrow 0$, thus $\left\||\varphi| f_{k}\right\|_{L^{2}} \nrightarrow$ 0 , that is $\int_{\mathbb{D}}|\varphi|^{2}\left|f_{k}\right|^{2} d A \nrightarrow 0$. But $T_{\varphi}$ is compact on $\mathcal{D}$, so for $F_{k}=\int_{0}^{z} f_{k} d w$, $\left\|T_{\varphi} F_{k}\right\|_{\mathcal{D}} \rightarrow 0$, further $\left\|T_{\varphi}^{*} T_{\varphi} F_{k}\right\|_{\mathcal{D}} \rightarrow 0$. Note $T_{\varphi}^{*}-T_{\bar{\varphi}} \in \mathcal{K}$, so $\left\|T_{|\varphi|^{2}} F_{k}\right\|_{\mathcal{D}} \rightarrow$ 0 (since $F_{k} \stackrel{w}{\rightarrow} 0$ in $\mathcal{D}$ and $\left\|F_{k}\right\|_{\mathcal{D}}=1$ ), thus $\left\langle T_{|\varphi|^{2}} F_{k}, F_{k}\right\rangle_{\frac{1}{2}} \rightarrow 0$, hence

$$
\left\langle\frac{\partial\left(|\varphi|^{2} F_{k}\right)}{\partial z}, F_{k}^{\prime}\right\rangle \rightarrow 0
$$

that is

$$
\left\langle\frac{\partial\left(|\varphi|^{2}\right)}{\partial z} F_{k}, F_{k}^{\prime}\right\rangle+\left\langle|\varphi|^{2} F_{k}^{\prime}, F_{k}^{\prime}\right\rangle \rightarrow 0
$$

Note

$$
\left\langle|\varphi|^{2} F_{k}^{\prime}, F_{k}^{\prime}\right\rangle=\left\langle|\varphi|^{2} f_{k}, f_{k}\right\rangle=\int_{\mathbb{D}}|\varphi|^{2}\left|f_{k}\right|^{2} d A \nrightarrow 0,
$$

and

$$
\left|\left\langle\frac{\partial\left(|\varphi|^{2}\right)}{\partial z} F_{k}, F_{k}^{\prime}\right\rangle\right| \leq\left\||\varphi|^{2}\right\|_{*}\left\|F_{k}\right\|_{L^{2}}\left\|f_{k}\right\|_{L^{2}} \rightarrow 0,
$$

hence $\left\langle T_{|\varphi|^{2}} F_{k}, F_{k}\right\rangle_{\frac{1}{2}} \nrightarrow 0$, this contradiction shows that $\left.\varphi\right|_{\partial \mathbb{D}} \equiv 0$.

Remark. If $\varphi \notin C^{1}(\overline{\mathbb{D}})$, then $T_{\varphi}$ may be non-compact even $\varphi \in C(\overline{\mathbb{D}})$ and $\left.\varphi\right|_{\partial \mathbb{D}}=0$. For instance, let $\varphi=\sqrt{1-|z|^{2}}$, then it is not difficult to check $T_{\varphi}$ is not a compact operator. In fact, $T_{\varphi}$ is also a unbounded operator on $\mathcal{D}$.

Theorem 6. Suppose $\varphi \in C^{1}(\overline{\mathbb{D}})$, then $\sigma_{e}\left(T_{\varphi}\right)=\varphi(\partial \mathbb{D})$.

Proof. Without loss of generality, assume $0 \in \varphi(\partial \mathbb{D})$, thus there is a $\zeta \in \partial \mathbb{D}$ such that $\varphi(\zeta)=0$. Write $f_{k}(z)=\left(\frac{1+z \bar{\zeta}}{2}\right)^{k}$, then $\frac{f_{k}}{\left\|f_{k}\right\|_{\mathcal{D}}} \stackrel{w}{\rightarrow} 0$, and $\frac{\left\|T_{\varphi} f_{k}\right\|_{\mathcal{D}}}{\left\|f_{k}\right\|_{\mathcal{D}}} \leq$ $\frac{\left\|\varphi f_{k}\right\|_{L^{2,1}}}{\left\|f_{k}\right\|_{\mathcal{D}}}$. Clearly

$$
\begin{aligned}
\left\|\varphi f_{k}\right\|_{L^{2,1}}^{2}= & \left\langle\frac{\partial \varphi}{\partial z} f_{k}+\varphi f_{k}^{\prime}, \frac{\partial \varphi}{\partial z} f_{k}+\varphi f_{k}^{\prime}\right\rangle+\left\langle\frac{\partial \varphi}{\partial \bar{z}} f_{k}, \frac{\partial \varphi}{\partial \bar{z}} f_{k}\right\rangle \\
= & \left\langle\frac{\partial \varphi}{\partial z} f_{k}, \frac{\partial \varphi}{\partial z} f_{k}\right\rangle+\left\langle\varphi f_{k}^{\prime}, \frac{\partial \varphi}{\partial z} f_{k}\right\rangle+\left\langle\frac{\partial \varphi}{\partial z} f_{k}, \varphi f_{k}^{\prime}\right\rangle \\
& +\left\langle\varphi f_{k}^{\prime}, \varphi f_{k}^{\prime}\right\rangle+\left\langle\frac{\partial \varphi}{\partial \bar{z}} f_{k}, \frac{\partial \varphi}{\partial \bar{z}} f_{k}\right\rangle
\end{aligned}
$$


note

$$
\frac{\left|\left\langle\frac{\partial \varphi}{\partial z} f_{k}, \frac{\partial \varphi}{\partial z} f_{k}\right\rangle+\left\langle\varphi f_{k}^{\prime}, \frac{\partial \varphi}{\partial z} f_{k}\right\rangle+\left\langle\frac{\partial \varphi}{\partial z} f_{k}, \varphi f_{k}^{\prime}\right\rangle+\left\langle\frac{\partial \varphi}{\partial z} f_{k}, \frac{\partial \varphi}{\partial \bar{z}} f_{k}\right\rangle\right|}{\left\|f_{k}\right\|_{\mathcal{D}}^{2}} \longrightarrow 0,
$$

and

$$
\begin{aligned}
\frac{\left\langle\varphi f_{k}^{\prime}, \varphi f_{k}^{\prime}\right\rangle}{\left\|f_{k}\right\|_{\mathcal{D}}^{2}} & =\frac{\int_{\mathbb{D}}|\varphi|^{2}\left|f_{k}^{\prime}\right|^{2} d A}{\int_{\mathbb{D}}\left|f_{k}^{\prime}\right|^{2} d A} \\
& =\frac{\int_{\mathbb{D}}|\varphi|^{2}\left|\left(\frac{1+z \bar{\zeta}}{2}\right)^{2(k-1)}\right| d A}{\int_{\mathbb{D}}\left|\left(\frac{1+z \bar{\zeta}}{2}\right)^{2(k-1)}\right| d A} \\
& =\frac{\int_{\mathbb{D}}|\varphi|^{2}\left|f_{k-1}\right|^{2} d A}{\int_{\mathbb{D}}\left|f_{k-1}\right|^{2} d A} \rightarrow|\varphi(\zeta)|^{2}=0
\end{aligned}
$$

(since $f_{k}$ is a peak function at $\zeta$ ). Thus $\frac{\left\|T_{\varphi} f_{k}\right\|_{\mathcal{D}}}{\left\|f_{k}\right\|_{\mathcal{D}}} \rightarrow 0$, this shows that $T_{\varphi}$ can not be Fredholm, hence $0 \in \sigma_{e}\left(T_{\varphi}\right)$. That is $\varphi(\partial \mathbb{D}) \subset \sigma_{e}\left(T_{\varphi}\right)$.

Conversely, if $0 \notin \varphi(\partial \mathbb{D})$, then $|\varphi(\zeta)|>\epsilon_{0}>0$ for any $\zeta \in \partial \mathbb{D}$, thus $\tilde{T}_{\varphi}$ is Fredholm on $L_{a}^{2}$, if $T_{\varphi}$ is not Fredholm on $\mathcal{D}$, then there is a sequence $\left\{F_{k}\right\} \subset \mathcal{D}$ with $\left\|F_{k}\right\|_{\mathcal{D}}=1, F_{k} \stackrel{w}{\rightarrow} 0$ such that $\left\|T_{\varphi} F_{k}\right\|_{\mathcal{D}} \rightarrow 0$, or $\left\|T_{\varphi}^{*} F_{k}\right\|_{\mathcal{D}} \rightarrow$ 0. Since $\tilde{T}_{\varphi}$ is Fredholm on $L_{a}^{2}$, there is an $S \in L\left(L_{a}^{2}\right)$ such that $\left[S T_{\varphi}\right]=$ $\left[T_{\varphi} S\right]=[I]$ in the Calkin algebra $\frac{L\left(L_{a}^{2}\right)}{\mathcal{K}}$, thus $S^{*} T_{\varphi}^{*}=I+K, K \in \mathcal{K}\left(L_{a}^{2}\right)$, assume $\left\|T_{\varphi}^{*} F_{k}\right\|_{\mathcal{D}} \rightarrow 0$. (For the case of $\left\|T_{\varphi} F_{k}\right\|_{\mathcal{D}} \rightarrow 0$, we can complete the proof similarly.) Write $f_{k}=F_{k}^{\prime}$, then $\left\|f_{k}\right\|_{L^{2}}=1$, and $f_{k} \stackrel{w}{\rightarrow} 0$ in $L_{a}^{2}$, thus $\left\langle S^{*} T_{\varphi}^{*} f_{k}, f_{k}\right\rangle \rightarrow 1$, that is $\left\langle T_{\varphi}^{*} f_{k}, S f_{k}\right\rangle \rightarrow 1$.

On the other hand,

$$
\begin{aligned}
\left\langle T_{\varphi}^{*} F_{k}, \int_{0}^{z}\left(S f_{k}\right)(w) d w\right\rangle_{\frac{1}{2}} & =\left\langle F_{k}, \varphi \int_{0}^{z}\left(S f_{k}\right)(w) d w\right\rangle_{\frac{1}{2}} \\
& =\left\langle f_{k}, \frac{\partial \varphi}{\partial z} \int_{0}^{z}\left(S f_{k}\right)(w) d w+\varphi S f_{k}\right\rangle \\
& =\left\langle f_{k}, \frac{\partial \varphi}{\partial z} \int_{0}^{z} S f_{k} d w\right\rangle+\left\langle f_{k}, \varphi S f_{k}\right\rangle .
\end{aligned}
$$

Since $S f_{k} \stackrel{w}{\rightarrow} 0$ in $L_{a}^{2},\left\|\int_{0}^{z} S f_{k} d w\right\|_{L_{a}^{2}} \rightarrow 0$, thus $\left\langle f_{k}, \frac{\partial \varphi}{\partial z} \int_{0}^{z} S f_{k}(w) d w\right\rangle \rightarrow 0$. Note $\left\langle f_{k}, \varphi S f_{k}\right\rangle=\left\langle T_{\varphi}^{*} f_{k}, S f_{k}\right\rangle \rightarrow 1 \neq 0$, this shows that $\left\langle T_{\varphi}^{*} F_{k}\right.$, $\left.\int_{0}^{z}\left(S f_{k}\right)(w) d w\right\rangle_{\frac{1}{2}} \rightarrow 1$, thus

$$
\left\|T_{\varphi}^{*} F_{k}\right\|_{\mathcal{D}}\|S\|_{L\left(L_{a}^{2}\right)} \geq\left\langle T_{\varphi}^{*} F_{k}, \int_{0}^{z} S f_{k} d w\right\rangle_{\frac{1}{2}} \rightarrow 1,
$$

this contradicts that $\left\|T_{\varphi}^{*} F_{k}\right\|_{\mathcal{D}} \rightarrow 0$. Hence $T_{\varphi}$ must be Fredholm, that is $0 \notin \sigma_{e}\left(T_{\varphi}\right)$. This follows the theorem. 
Lemma 7. For any $\varphi \in C^{1}(\overline{\mathbb{D}}),\left\|T_{\varphi}\right\|_{e}=\left\|\left[T_{\varphi}\right]\right\|=\left\|\left.\varphi\right|_{\partial \mathbb{D}}\right\|_{\infty}$, where $\left\|T_{\varphi}\right\|_{e}$ denotes the essential norm of $T_{\varphi}$.

Proof. Assume $\zeta \in \partial \mathbb{D}$ such that $|\varphi(\zeta)|=\left\|\left.\varphi\right|_{\partial \mathbb{D}}\right\|_{\infty}$, set $f_{\zeta}(z)=\frac{1+z \bar{\zeta}}{2}$, then $f_{k}(z)=\frac{f_{\zeta}^{k}}{\left\|f_{\zeta}^{k}\right\|_{\mathcal{D}}} \stackrel{w}{\rightarrow} 0$, thus for any $K \in \mathcal{K},\left\|K f_{k}\right\| \rightarrow 0$. Note

$$
\left|\left\langle T_{\varphi} f_{k}, f_{k}\right\rangle_{\frac{1}{2}}\right|=\left|\left\langle\frac{\partial \varphi}{\partial z} f_{k}, f_{k}^{\prime}\right\rangle+\left\langle\varphi f_{k}^{\prime}, f_{k}^{\prime}\right\rangle\right| \rightarrow|\varphi(\zeta)|=\left\|\left.\varphi\right|_{\partial \mathbb{D}}\right\|_{\infty}
$$

and $\left|\left\langle\left(T_{\varphi}+K\right) f_{k}, f_{k}\right\rangle_{\frac{1}{2}}\right| \leq\left\|T_{\varphi}+K\right\|$. So $\left\|\left.\varphi\right|_{\partial \mathbb{D}}\right\|_{\infty} \leq\left\|T_{\varphi}+K\right\|$ for any $K \in \mathcal{K}$. Hence $\left\|\left.\varphi\right|_{\partial \mathbb{D}}\right\|_{\infty} \leq\left\|T_{\varphi}\right\|_{e}$.

Conversely, by Lemma 1.2 in C.K. Fong [3], there is an orthogonal sequence $\left\{f_{k}\right\} \subset \mathcal{D}$ such that $\left\|T_{\varphi} f_{k}\right\| \rightarrow\left\|T_{\varphi}\right\|_{e}$, so for any $\epsilon>0$, there is a $k_{0}$ such that for any $k>k_{0},\left\|T_{\varphi} f_{k}\right\|>\left\|T_{\varphi}\right\|_{e}-\epsilon$. Since $\varphi \in C^{1}(\overline{\mathbb{D}})$, there is a $0<r<1$ such that $|\varphi(z)|<\left\|\left.\varphi\right|_{\partial \mathbb{D}}\right\|_{\infty}+\epsilon$ for any $|z|>r$. Note

$$
\begin{aligned}
\left\|T_{\varphi} f_{k}\right\|_{\mathcal{D}}^{2} \leq\left\|\varphi f_{k}\right\|_{L^{2,1}}^{2}= & \left\langle\varphi f_{k}, \varphi f_{k}\right\rangle_{\frac{1}{2}} \\
= & \left\langle\varphi f_{k}^{\prime}, \varphi f_{k}^{\prime}\right\rangle+\left\langle\frac{\partial \varphi}{\partial z} f_{k}, \varphi f_{k}^{\prime}\right\rangle \\
& +\left\langle\varphi f_{k}^{\prime}, \frac{\partial \varphi}{\partial z} f_{k}\right\rangle+\left\langle\frac{\partial \varphi}{\partial z} f_{k}, \frac{\partial \varphi}{\partial z} f_{k}\right\rangle \\
& +\left\langle\frac{\partial \varphi}{\partial \bar{z}} f_{k}, \frac{\partial \varphi}{\partial \bar{z}} f_{k}\right\rangle
\end{aligned}
$$

clearly,

$$
\left\langle\frac{\partial \varphi}{\partial z} f_{k}, \varphi f_{k}^{\prime}\right\rangle+\left\langle\varphi f_{k}^{\prime}, \frac{\partial \varphi}{\partial z} f_{k}\right\rangle+\left\langle\frac{\partial \varphi}{\partial z} f_{k}, \frac{\partial \varphi}{\partial z} f_{k}\right\rangle+\left\langle\frac{\partial \varphi}{\partial \bar{z}} f_{k}, \frac{\partial \varphi}{\partial \bar{z}} f_{k}\right\rangle \rightarrow 0,
$$

so there is a $k_{1}$ such that for any $k>k_{1},\left\|T_{\varphi} f_{k}\right\|^{2} \leq\left|\left\langle\varphi f_{k}^{\prime}, \varphi f_{k}^{\prime}\right\rangle\right|+\epsilon$. Since $f_{k} \stackrel{w}{\rightarrow} 0$, we know that $f_{k} \stackrel{\text { uniformly }}{\longrightarrow} 0$ on any compact subset of $\mathbb{D}$, so for any $0<t<1, \int_{\{|z| \leq t\}}\left|f_{k}\right|^{2} d A \rightarrow 0(k \rightarrow \infty)$, assume $f_{k}(z)=\sum_{i=1}^{\infty} a_{n}^{(k)} z^{n}$, thus

$$
\sum_{i=1}^{\infty} a_{n}^{(k)} \frac{t^{2(n+1)}}{n+1}=\int_{\{|z| \leq t\}}\left|f_{k}\right|^{2} d A \rightarrow 0 .
$$

Without loss of generality, assume $t>r$, thus

$$
\int_{\{|z| \leq r\}}\left|f_{k}^{\prime}\right|^{2} d A=\sum_{i=1}^{\infty}\left|a_{n}^{(k)}\right|^{2} n r^{2 n}=\sum_{i=1}^{\infty}\left|a_{n}^{(k)}\right|^{2} \frac{t^{2(n+1)}}{n+1} n(n+1) t^{-2}\left(\frac{r}{t}\right)^{2 n}
$$


since $\left(\frac{r}{t}\right)^{2 n} n(n+1) t^{-2} \rightarrow 0(n \rightarrow \infty)$, it is clearly that $\int_{\{|z| \leq r\}}\left|f_{k}^{\prime}\right|^{2} d A \rightarrow 0$, so there is a $k_{2}$ such that for any $k>k_{2}, \int_{\{|z| \leq r\}}\left|f_{k}^{\prime}\right|^{2} d A<\epsilon$, consequently,

$$
\begin{aligned}
\left|\left\langle\varphi f_{k}^{\prime}, \varphi f_{k}^{\prime}\right\rangle\right| & =\int_{\mathbb{D}}|\varphi|^{2}\left|f_{k}^{\prime}\right|^{2} d A \\
& =\int_{\{|z| \leq r\}}|\varphi|^{2}\left|f_{k}^{\prime}\right|^{2} d A+\int_{\{|z|>r\}}|\varphi|^{2}\left|f_{k}^{\prime}\right|^{2} d A \\
& \leq\|\varphi\|_{\infty}^{2} \int_{\{|z| \leq r\}}\left|f_{k}^{\prime}\right|^{2} d A+\left(\left\|\left.\varphi\right|_{\partial \mathbb{D}}\right\|_{\infty}+\epsilon\right)^{2} \int_{\{|z|>r\}}\left|f_{k}^{\prime}\right|^{2} d A \\
& \leq\|\varphi\|_{\infty}^{2} \int_{\{|z| \leq r\}}\left|f_{k}^{\prime}\right|^{2} d A+\left(\left\|\left.\varphi\right|_{\partial \mathbb{D}}\right\|_{\infty}+\epsilon\right)^{2} \\
& <\|\varphi\|_{\infty}^{2} \epsilon+\left(\left\|\left.\varphi\right|_{\partial \mathbb{D}}\right\|_{\infty}+\epsilon\right)^{2} .
\end{aligned}
$$

Furthermore, for any $k>\max \left\{k_{0}, k_{1}, k_{2}\right\}$

$$
\begin{aligned}
\left\|T_{\varphi} f_{k}\right\|^{2} & \leq\|\varphi\|_{\infty}^{2} \int_{\{|z| \leq r\}}\left|f_{k}^{\prime}\right|^{2} d A+\left(\left\|\left.\varphi\right|_{\partial \mathbb{D}}\right\|_{\infty}+\epsilon\right)^{2}+\epsilon \\
& \leq\left(\|\varphi\|_{\infty}^{2}+1\right) \epsilon+\left(\left\|\left.\varphi\right|_{\partial \mathbb{D}}\right\|_{\infty}+\epsilon\right)^{2} .
\end{aligned}
$$

Hence $\lim _{k \rightarrow \infty}\left\|T_{\varphi} f_{k}\right\| \leq\left\|\left.\varphi\right|_{\partial \mathbb{D}}\right\|_{\infty}$ by the arbitrarity of $\epsilon$. This shows that $\left\|T_{\varphi}\right\|_{e} \leq\left\|\left.\varphi\right|_{\partial \mathbb{D}}\right\|_{\infty}$. We are done.

Theorem 8. Let $\mathcal{I}=\mathcal{I}\left(C^{1}\right)$ be the $C^{*}$-algebra generated by $\left\{T_{\varphi} \mid \varphi \in C^{1}(\overline{\mathbb{D}})\right\}$, then the commutator ideal $\mathcal{C}\left(C^{1}\right)$ of $\mathcal{I}$ equals $\mathcal{K}(\mathcal{D})$, and $\frac{\mathcal{I}}{\mathcal{K}} \cong C(\partial \mathbb{D})$. Consequently, following short sequence

$$
0 \rightarrow \mathcal{K} \rightarrow \mathcal{I} \rightarrow C(\partial \mathbb{D}) \rightarrow 0
$$

is exact.

Proof. By Proposition 3, we know that $\mathcal{C}\left(C^{1}\right) \subset \mathcal{K}$, since $\mathcal{K}$ is minimal, $\mathcal{C}\left(C^{1}\right)=\mathcal{K}$. By Lemma $4,\left[T_{\varphi}^{*}\right]=\left[T_{\bar{\varphi}}\right]$ in $\frac{\mathcal{I}}{\mathcal{K}}$. Define $\xi:\left\{\left[T_{\varphi}\right] \mid \varphi \in C^{1}(\overline{\mathbb{D}})\right\} \rightarrow$ $C(\partial \mathbb{D})$ as $\xi\left(\left[T_{\varphi}\right]\right)=\left.\varphi\right|_{\partial \mathbb{D}}$, it is easy to see that $\xi$ is well-defined, and one-toone by Proposition 5. By Proposition 3, Lemma 4 and Lemma 7, we see that $\xi$ is an isometric ${ }^{*}$-homomorphism. Hence $\xi$ can be extended to $\frac{\mathcal{I}}{\mathcal{K}}$, in fact, for any $[T] \in \frac{\mathcal{I}}{\mathcal{K}}$, there is a sequence $\left[T_{\varphi_{k}}\right]$ such that $\left\|\left[T_{\varphi_{k}}\right]-[T]\right\| \rightarrow 0$, thus $\left\|\left.\left(\varphi_{k}-\varphi_{j}\right)\right|_{\partial \mathbb{D}}\right\|_{\infty}=\left\|\left[T_{\varphi_{k}-\varphi_{j}}\right]\right\|=\left\|\left[T_{\varphi_{k}}\right]-\left[T_{\varphi_{j}}\right]\right\| \rightarrow 0$. Hence there is a $\varphi \in$ $C(\partial \mathbb{D})$ such that $\left\|\left.\varphi_{k}\right|_{\partial \mathbb{D}}-\varphi\right\|_{\infty} \rightarrow 0$. Let $\xi([T])=\varphi$, then $\xi$ is well-defined on $\frac{\mathcal{I}}{\mathcal{K}}$, and $\|\xi([T])\|_{\infty}=\|\varphi\|_{\infty}=\lim _{k \rightarrow \infty}\left\|\left.\varphi_{k}\right|_{\partial \mathbb{D}}\right\|_{\infty}=\lim _{k \rightarrow \infty}\left\|\left[T_{\varphi_{k}}\right]\right\|=\|[T]\|$, so $\xi$ is an isometry from $\frac{\mathcal{I}}{\mathcal{K}}$ into $C(\partial \mathbb{D})$. For any $\varphi \in C(\partial \mathbb{D})$, there is a polynomial sequence $\left\{p_{k}\right\} \subset C^{1}(\overline{\mathbb{D}})$ such that $\left\|\left.p_{k}\right|_{\partial \mathbb{D}}-\varphi\right\|_{\infty} \rightarrow 0$, thus $\left\|\left.\left(p_{k}-p_{j}\right)\right|_{\partial \mathbb{D}}\right\|_{\infty} \rightarrow 0$. Furthermore, $\left\|\left[T_{p_{k}}\right]-\left[T_{p_{j}}\right]\right\| \rightarrow 0$, so there is a $[T] \in \frac{\mathcal{I}}{\mathcal{K}}$ such that $\left\|\left[T_{p_{k}}\right]-[T]\right\| \rightarrow 0$, hence $\xi([T])=\varphi$, i.e. $\xi$ is a surjection onto $C(\partial \mathbb{D})$. This shows that $\xi$ is a ${ }^{*}$-isomorphism between $\frac{\mathcal{I}}{\mathcal{K}}$ and $C(\partial \mathbb{D})$. The proof is thus completed. 


\section{Toeplitz operators with symbols in $H_{1}^{\infty}+C^{1}(\overline{\mathbb{D}})$.}

It is well-known that $H^{\infty}+C(\partial \mathbb{D})$ is a norm-closed algebra (c.f. R.G. Douglas [2]), Rudin [6] proved that $H^{\infty}\left(\partial B_{n}\right)+C\left(\partial B_{n}\right)$ is also a normclosed algebra, where $B_{n}$ is the unit ball of $\mathbb{C}^{n}$. The Toeplitz operators with symbols in $H^{\infty}+C$ on Hardy or Bergman spaces have many important properties, their essential spectra and Fredholm index can be completely determined by their symbols. In this section, we prove that $H_{1}^{\infty}+C^{1}(\overline{\mathbb{D}})$ is a norm-closed space relative to a suitable norm, and obtain the representation of essential spectra of the Toeplitz operators with symbols in $H_{1}^{\infty}+C^{1}(\overline{\mathbb{D}})$.

For any $\varphi \in H_{1}^{\infty}+C^{1}(\overline{\mathbb{D}})$, define

$$
\|\varphi\|_{*}=\sup _{z \in \mathbb{D}} \max \left\{|\varphi|,\left|\frac{\partial \varphi}{\partial z}\right|,\left|\frac{\partial \varphi}{\partial \bar{z}}\right|\right\}
$$

it is clearly that $\|\cdot\|_{*}$ is a norm on $H_{1}^{\infty}+C^{1}(\overline{\mathbb{D}})$.

Theorem 9. $H_{1}^{\infty}+C^{1}(\overline{\mathbb{D}})$ is a closed space relative to the norm $\|\cdot\|_{*}$.

Proof. Our proof is similar to that of Rudin [6]. Assume $\varphi \in \operatorname{cl}\left(H_{1}^{\infty}+\right.$ $C^{1}(\overline{\mathbb{D}})$ ), to prove $\varphi \in H_{1}^{\infty}+C^{1}(\overline{\mathbb{D}})$, we first prove that for any $\psi \in H_{1}^{\infty}+$ $C^{1}(\overline{\mathbb{D}})$, there are $\psi_{1} \in H_{1}^{\infty}$, and $\psi_{2} \in C^{1}(\overline{\mathbb{D}})$ such that $\psi=\psi_{1}+\psi_{2}$ and $\left\|\psi_{1}\right\|_{*} \leq 3\|\psi\|_{*},\left\|\psi_{2}\right\|_{*} \leq 2\|\psi\|_{*}$. In fact, if $\psi=\tilde{\psi}_{1}+\tilde{\psi}_{2} \in \tilde{\sim}_{1}^{\infty}+C^{1}(\overline{\mathbb{D}})$, $\tilde{\psi}_{1} \in H_{1}^{\infty}, \tilde{\psi}_{2} \in C^{1}(\overline{\mathbb{D}})$, then write $\tilde{\psi}_{2}^{r}(z)=\tilde{\psi}_{2}(r z)$, clearly, $\left\|\tilde{\psi}_{2}^{r}-\tilde{\psi}_{2}\right\|_{\infty} \rightarrow$ $0\left(r \rightarrow 1^{-}\right)$, and $\frac{\partial \tilde{\psi}_{2}^{r}}{\partial z}(z)=r \frac{\partial \tilde{\psi}_{2}}{\partial z}(r z), \frac{\partial \tilde{\psi}_{2}^{r}}{\partial \bar{z}}(z)=r \frac{\partial \tilde{\psi}_{2}}{\partial \bar{z}}(r z)$, so $\left\|\tilde{\psi}_{2}^{r}-\tilde{\psi}_{2}\right\|_{*} \rightarrow 0$. Fix a $r_{0}$ such that $\left\|\tilde{\psi}_{2}^{r_{0}}-\tilde{\psi}_{2}\right\|_{*} \leq\|\psi\|_{*}$. Set

$$
\psi_{2}=\tilde{\psi}_{2}-\tilde{\psi}_{2}^{r_{0}}+\psi^{r_{0}}, \psi_{1}=\tilde{\psi}_{1}-\tilde{\psi}_{1}^{r_{0}},
$$

then $\psi=\psi_{1}+\psi_{2}$, and $\left\|\psi_{1}\right\|_{*}=\left\|\psi-\psi_{2}\right\|_{*} \leq 3\|\psi\|_{*},\left\|\psi_{2}\right\|_{*} \leq 2\|\psi\|_{*}$. Since $\varphi \in \operatorname{cl}\left(H_{1}^{\infty}+C^{1}(\overline{\mathbb{D}})\right)$, there are $\varphi_{i} \in H_{1}^{\infty}+C^{1}(\overline{\mathbb{D}})$, such that $\left\|\varphi_{i}\right\|_{*} \leq$ $2^{-i}(i \geq 2)$, and $\varphi=\sum_{i=1}^{\infty} \varphi_{i}$ (in fact, there is a sequence $\left\{p_{k}\right\} \subset H_{1}^{\infty}+$ $C^{1}(\overline{\mathbb{D}})$ such that $\left\|p_{k}-\varphi\right\|_{*} \rightarrow 0$, thus there is a subsequence $\left\{p_{k}\right\}$ such that $\left\|p_{k_{i+1}}-p_{k_{i}}\right\|_{*}<\frac{1}{2^{i}}$, write $\varphi_{1}=p_{k_{1}}, \varphi_{i}=p_{k_{i+1}}-p_{k_{i}}(i \geq 2)$, then $\sum_{i=1}^{\infty} \varphi_{i}=\varphi$ and $\left.\left\|\varphi_{i}\right\|_{*}<\frac{1}{2^{i}}(i \geq 2)\right)$. For each $\varphi_{i}$, there is a $\varphi_{i}^{(1)} \in H_{1}^{\infty}$, $\varphi_{i}^{(2)} \in C^{1}(\overline{\mathbb{D}})$ such that $\left\|\varphi_{i}^{(1)}\right\|_{*} \leq 3\left\|\varphi_{i}\right\|_{*},\left\|\varphi_{i}^{(2)}\right\|_{*} \leq 2\left\|\varphi_{i}\right\|_{*}, \varphi_{i}=\varphi_{i}^{(1)}+\varphi_{i}^{(2)}$, thus $\varphi^{(1)}=\sum_{i=1}^{\infty} \varphi_{i}^{(1)} \in H_{1}^{\infty} \quad\left(\right.$ since $H_{1}^{\infty}$ is closed relative to $\left.\|\cdot\|_{*}\right), \varphi^{(2)}=$ $\sum_{i=1}^{\infty} \varphi_{i}^{(2)} \in C^{1}(\overline{\mathbb{D}})$, and $\varphi=\varphi^{(1)}+\varphi^{(2)}$. That is $\varphi \in H_{1}^{\infty}+C^{1}(\overline{\mathbb{D}})$.

Proposition 10. If $\varphi \in H_{1}^{\infty}+C^{1}(\overline{\mathbb{D}})$ satisfies $\left.\varphi\right|_{\partial \mathbb{D}}=0$, then $T_{\varphi}$ is a compact operator on $\mathcal{D}$, where $\left.\varphi\right|_{\partial \mathbb{D}}$ denotes the radial boundary values of $\varphi$.

Proof. Suppose $\varphi=\varphi_{1}+\varphi_{2}, \varphi_{1} \in H_{1}^{\infty}, \varphi_{2} \in C^{1}(\overline{\mathbb{D}})$, since $\left.\varphi\right|_{\partial \mathbb{D}}=0,\left.\varphi_{1}\right|_{\partial \mathbb{D}}=$ $-\left.\varphi_{2}\right|_{\partial \mathbb{D}}$, thus $\left.\varphi_{1}\right|_{\partial \mathbb{D}} \in C(\partial \mathbb{D})$, further $\varphi_{1}(z)=P\left[\left.\varphi_{1}\right|_{\partial \mathbb{D}}\right] \in H_{1}^{\infty} \cap A(\mathbb{D})$ (where $P\left[\left.\varphi\right|_{\partial \mathbb{D}}\right]$ denotes the Poisson integral of $\left.\left.\varphi\right|_{\partial \mathbb{D}}\right)$, consequently, $\varphi \in$ $C(\overline{\mathbb{D}}) \cap\left(H_{1}^{\infty}+C^{1}(\overline{\mathbb{D}})\right)$. 
Suppose $\left\{f_{k}\right\} \subset \mathcal{D},\left\|f_{k}\right\|_{\mathcal{D}}=1, f_{k} \stackrel{w}{\rightarrow} 0$, then

$$
\begin{aligned}
\left\|T_{\varphi} f_{k}\right\|_{\mathcal{D}}^{2} \leq & \left\|\varphi f_{k}\right\|_{L^{2,1}}^{2}=\left\langle\varphi f_{k}, \varphi f_{k}\right\rangle_{\frac{1}{2}} \\
= & \left\langle\frac{\partial \varphi}{\partial z} f_{k}+f_{k}^{\prime} \varphi, \frac{\partial \varphi}{\partial z} f_{k}+f_{k}^{\prime} \varphi\right\rangle+\left\langle\frac{\partial \varphi}{\partial \bar{z}} f_{k}, \frac{\partial \varphi}{\partial \bar{z}} f_{k}\right\rangle \\
= & \left\|\frac{\partial \varphi}{\partial z} f_{k}\right\|_{L^{2}}^{2}+2 \operatorname{Re} \int_{\mathbb{D}} f_{k}^{\prime} \varphi \frac{\overline{\partial \varphi}}{\partial z} \overline{f_{k}} d A+\left\|\varphi f_{k}^{\prime}\right\|_{L^{2}}^{2}+\left\|\frac{\partial \varphi}{\partial \bar{z}} f_{k}\right\|_{L^{2}}^{2} \\
\leq & \|\varphi\|_{*}^{2}\left\|f_{k}\right\|_{L^{2}}^{2}+2\|\varphi\|_{*}^{2}\left\|f_{k}\right\|_{\mathcal{D}}\left\|f_{k}\right\|_{L^{2}} \\
& +\left\|\varphi f_{k}^{\prime}\right\|_{L^{2}}^{2}+\|\varphi\|_{*}^{2}\left\|f_{k}\right\|_{L^{2}}^{2} .
\end{aligned}
$$

Since $\left\|f_{k}\right\|_{\mathcal{D}}=1$ and $f_{k} \stackrel{w}{\rightarrow} 0$ in $\mathcal{D},\left\|f_{k}\right\|_{L^{2}} \rightarrow 0$, so

$$
\|\varphi\|_{*}^{2}\left\|f_{k}\right\|_{L^{2}}^{2}+2\|\varphi\|_{*}^{2}\left\|f_{k}\right\|_{\mathcal{D}}\left\|f_{k}\right\|_{L^{2}}+\|\varphi\|_{*}^{2}\left\|f_{k}\right\|_{L^{2}}^{2} \rightarrow 0 .
$$

Note $\varphi \in C(\overline{\mathbb{D}})$, and $\left.\varphi\right|_{\partial \mathbb{D}}=0$, hence for any $\epsilon>0$, there is a $0<\delta<1$ such that $|\varphi(z)|<\epsilon$ for any $|z|>\delta$, thus

$$
\begin{aligned}
\int_{\mathbb{D}}|\varphi|^{2}\left|f_{k}^{\prime}\right|^{2} d A & \leq \epsilon^{2} \int_{\{|z|>\delta\}}\left|f_{k}^{\prime}\right|^{2} d A+\int_{\{|z| \leq \delta\}}|\varphi|^{2}\left|f_{k}^{\prime}\right|^{2} d A \\
& \leq \epsilon^{2}+\|\varphi\|_{*}^{2} \int_{\{|z| \leq \delta\}}\left|f_{k}^{\prime}\right|^{2} d A .
\end{aligned}
$$

By $f_{k} \stackrel{w}{\rightarrow} 0$, we know that $\int_{\{|z| \leq \delta\}}\left|f_{k}^{\prime}\right|^{2} d A \rightarrow 0$, hence $\int_{\mathbb{D}}|\varphi|^{2}\left|f_{k}^{\prime}\right|^{2} d A \rightarrow$ $0(k \rightarrow \infty)$, furthermore $\left\|T_{\varphi} f_{k}\right\|_{\mathcal{D}}^{2} \rightarrow 0$, this shows that $T_{\varphi}$ is compact on $\mathcal{D}$.

Proposition 11. If $\varphi \in H_{1}^{\infty}(\mathbb{D})$, then on Dirichlet space $\mathcal{D}, \sigma\left(T_{\varphi}\right)=\overline{\varphi(\mathbb{D})}$.

Proof. Note for any $f \in \mathcal{D}$,

$$
\left\langle f, T_{\varphi}^{*} K\right\rangle_{\frac{1}{2}}=\left\langle T_{\varphi} f, K\right\rangle_{\frac{1}{2}}=\langle\varphi f, K\rangle_{\frac{1}{2}}=\varphi(z) f(z),
$$

so $T_{\varphi}^{*} K(z, w)=\overline{\varphi(z)} K(z, w)$, this shows that $\overline{\varphi(\mathbb{D})} \subset \sigma\left(T_{\varphi}\right)$.

Conversely, if $0 \notin \overline{\varphi(\mathbb{D})}$, then there is a $\delta>0$ such that $|\varphi(z)|>\delta>$ $0(\forall z \in \mathbb{D})$, thus $\varphi^{-1} \in H^{\infty}$. Note

$$
\left|\frac{\partial\left(\varphi^{-1}\right)}{\partial z}\right|=\left|-\frac{\varphi^{\prime}}{\varphi^{2}}\right| \leq \frac{1}{\delta^{2}}\left|\varphi^{\prime}\right| \leq \frac{1}{\delta^{2}}\|\varphi\|_{*}<\infty
$$

we have $\varphi^{-1} \in H_{1}^{\infty}$, so $T_{\varphi^{-1}} T_{\varphi}=T_{\varphi} T_{\varphi^{-1}}=I$, that is $T_{\varphi}$ is invertible, hence $\sigma\left(T_{\varphi}\right) \subset \overline{\varphi(\mathbb{D})}$. The proof is thus complete.

Theorem 12. If $\varphi=\varphi_{1}+\varphi_{2} \in H_{1}^{\infty}+C^{1}(\overline{\mathbb{D}})$, where $\varphi_{1} \in H_{1}^{\infty}, \varphi_{2} \in C^{1}(\overline{\mathbb{D}})$, then on Dirichlet space $\mathcal{D}$,

$$
\sigma_{e}\left(T_{\varphi}\right)=\overline{\cap_{0<\delta<1}\{\varphi(z)|| z \mid>\delta\}}=\varphi(\partial \mathbb{D}) .
$$


Proof. Without loss of generality, assume $0 \in \overline{\cap_{0<\delta<1}\{\varphi(z)|| z \mid>\delta\}}$, then there is a sequence $\left\{z_{k}\right\} \subset \mathbb{D}$ with $z_{n} \rightarrow \zeta \in \partial \mathbb{D}$, such that $\varphi\left(z_{n}\right) \rightarrow 0$, thus $\tilde{T}_{\varphi}$ is not Fredholm on $L_{a}^{2}$ (c.f. G.F. Cao [1] or MacDonald [4]), hence there is a sequence $\left\{f_{k}\right\} \subset L_{a}^{2}$ with $\left\|f_{k}\right\|_{L^{2}}=1, f_{k} \stackrel{w}{\rightarrow} 0$ such that $\left\|\tilde{T}_{\varphi} f_{k}\right\|_{L^{2}} \rightarrow 0$ or $\left\|\tilde{T}_{\varphi}^{*} f_{k}\right\|_{L^{2}}=\left\|\tilde{T}_{\bar{\varphi}} f_{k}\right\|_{L^{2}} \rightarrow 0$. If $T_{\varphi}$ is Fredholm on $\mathcal{D}$, then there is a bounded operator $S$ on $\mathcal{D}$ such that $S T_{\varphi}-I, T_{\varphi} S-I \in \mathcal{K}(\mathcal{D})$, further $S^{*} T_{\varphi}^{*}-I, T_{\varphi}^{*} S^{*}-I \in \mathcal{K}(\mathcal{D})$. Write $F_{k}(z)=\int_{0}^{z} f_{k}(w) d w$, then $F_{k} \in \mathcal{D}$ and $\left\|F_{k}\right\|_{\mathcal{D}}=\left\|f_{k}\right\|_{L^{2}}=1$, so $\left\|F_{k}\right\|_{L^{2}} \rightarrow 0$. In addition, it is clearly that $F_{k} \stackrel{w}{\rightarrow} 0$ in $\mathcal{D}$. Thus

$$
\begin{aligned}
\lim _{k \rightarrow \infty}\left\langle S T_{\varphi} F_{k}, F_{k}\right\rangle_{\frac{1}{2}} & =\lim _{k \rightarrow \infty}\left\langle T_{\varphi} S F_{k}, F_{k}\right\rangle_{\frac{1}{2}} \\
& =\lim _{k \rightarrow \infty}\left\langle T_{\varphi}^{*} S^{*} F_{k}, F_{k}\right\rangle_{\frac{1}{2}} \\
& =\lim _{k \rightarrow \infty}\left\langle S^{*} T_{\varphi}^{*} F_{k}, F_{k}\right\rangle_{\frac{1}{2}}=1 .
\end{aligned}
$$

Hence, without loss of generality, we can assume that $\left\|\tilde{T}_{\varphi}^{*} f_{k}\right\|_{L^{2}} \rightarrow 0$ (similarly for the case of $\left.\left\|\tilde{T}_{\varphi} f_{k}\right\|_{L^{2}} \rightarrow 0\right)$. Set $G_{k}=S F_{k}$, then $\left\|G_{k}\right\|_{\mathcal{D}} \leq\|S\|\left\|F_{k}\right\|_{\mathcal{D}}=$ $\|S\|$, and $G_{k} \stackrel{w}{\rightarrow} 0$, so $\left\|G_{k}\right\|_{L^{2}} \rightarrow 0$, and

$$
\begin{aligned}
\left|\left\langle T_{\varphi} S F_{k}, F_{k}\right\rangle_{\frac{1}{2}}\right| & =\left|\left\langle\varphi\left(S F_{k}\right)^{\prime}, f_{k}\right\rangle+\left\langle\frac{\partial \varphi}{\partial z} S F_{k}, f_{k}\right\rangle\right| \\
& \leq\left|\left\langle\left(S F_{k}\right)^{\prime}, \tilde{T}_{\varphi}^{*} f_{k}\right\rangle\right|+\|\varphi\|_{*}\left\|S F_{k}\right\|_{L^{2}}\left\|f_{k}\right\|_{L^{2}} \\
& \leq\left\|\left(S F_{k}\right)^{\prime}\right\|_{L^{2}}\left\|\tilde{T}_{\varphi}^{*} f_{k}\right\|_{L^{2}}+\|\varphi\|_{*}\left\|G_{k}\right\|_{L^{2}}\left\|f_{k}\right\|_{L^{2}} \\
& =\left\|G_{k}\right\|_{\mathcal{D}}\left\|\tilde{T}_{\varphi}^{*} f_{k}\right\|_{L^{2}}+\|\varphi\|_{*}\left\|G_{k}\right\|_{L^{2}}\left\|f_{k}\right\|_{L^{2}} \rightarrow 0 .
\end{aligned}
$$

This contradicts that $\left\langle T_{\varphi} S F_{k}, F_{k}\right\rangle_{\frac{1}{2}} \rightarrow 1$. hence $T_{\varphi}$ must be non-Fredholm. That is $0 \in \sigma_{e}\left(T_{\varphi}\right)$, consequently $\frac{2}{\cap_{0<\delta<1}\{\varphi(z)|| z \mid>\delta\}} \subset \sigma_{e}\left(T_{\varphi}\right)$.

Conversely, assume $0 \notin \overline{\cap_{0<\delta<1}\{\varphi(z)|| z \mid>\delta\}}$, thus there are $\epsilon, \delta>0$ such that $|\varphi(z)|>\epsilon$ for any $|z|>\delta$, we prove that $T_{\varphi}$ is Fredholm on $\mathcal{D}$. Otherwise, there is a sequence $\left\{F_{k}\right\} \subset \mathcal{D}$ with $\left\|F_{k}\right\|_{\mathcal{D}}=1, F_{k} \stackrel{w}{\rightarrow} 0$, such that $\left\|T_{\varphi} F_{k}\right\|_{\mathcal{D}} \rightarrow 0$ or $\left\|T_{\varphi}^{*} F_{k}\right\|_{\mathcal{D}} \rightarrow 0$. Similar to above proof, we can assume $\left\|T_{\varphi}^{*} F_{k}\right\|_{\mathcal{D}} \rightarrow 0$ (if $\left\|T_{\varphi} F_{k}\right\|_{\mathcal{D}} \rightarrow 0$, the proof will be simpler). It is well-known that $\stackrel{\sim}{\rightarrow} T_{\varphi}$ is Fredholm on $L_{a}^{2}$ if $|\varphi(z)|>\epsilon$ for any $|z|>\delta$, hence there is a $S \in L\left(L_{a}^{2}\right)$ such that $S \tilde{T}_{\varphi}-I, \tilde{T}_{\varphi} S-I \in \mathcal{K}\left(L_{a}^{2}\right)$, further, $S^{*} \tilde{T}_{\varphi}^{*}-I, \tilde{T}_{\varphi}^{*} S^{*}-I \in \mathcal{K}\left(L_{a}^{2}\right)$. Set $f_{k}=F_{k}^{\prime}, g_{k}=S f_{k}$, then $f_{k} \stackrel{w}{\rightarrow} 0, g_{k} \stackrel{w}{\rightarrow} 0$ in $L_{a}^{2}$ and $\left\|f_{k}\right\|_{L_{a}^{2}}=\left\|F_{k}\right\|_{\mathcal{D}}=1,\left\|g_{k}\right\|_{L_{a}^{2}} \leq\|S\|\left\|f_{k}\right\|_{L_{a}^{2}}=\|S\|$, so $\left\langle S^{*} \tilde{T}_{\varphi}^{*} f_{k}, f_{k}\right\rangle \rightarrow$ 
1, that is $\left\langle\tilde{T}_{\varphi}^{*} f_{k}, S f_{k}\right\rangle \rightarrow 1$. Note

$$
\begin{aligned}
\left\langle T_{\varphi}^{*} F_{k}, \int_{0}^{z} S f_{k}(w) d w\right\rangle_{\frac{1}{2}} & =\left\langle F_{k}, \varphi \int_{0}^{z} S f_{k} d w\right\rangle_{\frac{1}{2}} \\
& =\left\langle f_{k},\left(\frac{\partial \varphi}{\partial z} \int_{0}^{z} S f_{k} d w+\varphi S f_{k}\right)\right\rangle_{L^{2}} \\
& =\left\langle f_{k}, \frac{\partial \varphi}{\partial z} \int_{0}^{z} g_{k} d w\right\rangle+\left\langle f_{k}, \varphi g_{k}\right\rangle
\end{aligned}
$$

and $g_{k} \stackrel{w}{\rightarrow} 0$, so $\left\|\int_{0}^{z} g_{k} d w\right\|_{L^{2}} \rightarrow 0$, and

$$
\left|\left\langle f_{k}, \frac{\partial \varphi}{\partial z} \int_{0}^{z} g_{k} d w\right\rangle\right| \leq\left\|f_{k}\right\|_{L_{a}^{2}}\|\varphi\|_{*}\left\|\int_{0}^{z} g_{k} d w\right\|_{L^{2}} \rightarrow 0
$$

but $\left\langle f_{k}, \varphi g_{k}\right\rangle=\left\langle\tilde{T}_{\varphi}^{*} f_{k}, S f_{k}\right\rangle \rightarrow 1 \neq 0$, hence $\left\langle T_{\varphi}^{*} F_{k}, \int_{0}^{z} g_{k} d w\right\rangle_{\frac{1}{2}} \rightarrow 1 \neq 0$, this contradicts that $\left\|T_{\varphi}^{*} F_{k}\right\|_{\mathcal{D}} \rightarrow 0$. It shows that $T_{\varphi}$ must be Fredholm on $\mathcal{D}$. That is $0 \notin \sigma_{e}\left(T_{\varphi}\right)$. Note the functions with derivatives in $H^{\infty}$ are continuous in the closed unit disk, hence $H_{1}^{\infty}+C^{1}(\overline{\mathbb{D}}) \subset C(\overline{\mathbb{D}})$, consequently, $\overline{\cap_{0<\delta<1}\{\varphi(z)|| z \mid>\delta\}}=\varphi(\partial \mathbb{D})$. We are done.

Remark. If $\varphi \in H^{\infty}$, then $T_{\bar{\varphi}}$ is always bounded on $\mathcal{D}$, in fact, for any $f, g \in \mathcal{D}$,

$$
\begin{aligned}
\left|\left\langle T_{\bar{\varphi}} f, g\right\rangle_{\frac{1}{2}}\right| & =\left|\langle\bar{\varphi} f, g\rangle_{\frac{1}{2}}\right|=\left|\left\langle\bar{\varphi} f^{\prime}, g^{\prime}\right\rangle\right| \\
& =\left|\left\langle\tilde{T}_{\bar{\varphi}} f^{\prime}, g^{\prime}\right\rangle\right| \leq\left\|\tilde{T}_{\bar{\varphi}}\right\|\left\|f^{\prime}\right\|_{L^{2}}\left\|g^{\prime}\right\|_{L^{2}} \leq\|\varphi\|_{\infty}\|f\|_{\mathcal{D}}\|g\|_{\mathcal{D}},
\end{aligned}
$$

hence $\left\|T_{\bar{\varphi}} f\right\| \leq\|\varphi\|_{\infty}\|f\|_{\mathcal{D}}$. However, for $\varphi \in H^{\infty}, T_{\varphi}$ may be unbounded on $\mathcal{D}$. Let

$$
L_{1}^{\infty, 1}=\left\{f \in L^{2,1} \mid f, \frac{\partial f}{\partial z} \in L^{\infty}(\mathbb{D}, d A)\right\},
$$

then $L_{1}^{\infty, 1} \cup \overline{H^{\infty}}$ is perhaps the most suitable symbol space of Toeplitz operators which are bounded. Also, we can prove that if $\varphi \in L_{1}^{\infty, 1}$ has a compact support set, then $T_{\varphi}$ is compact on $\mathcal{D}$.

\section{An index formula of Toeplitz operators.}

The classical index formula shows that for $\varphi \in C(\partial \mathbb{D})$, if $|\varphi|>\epsilon>0$, then $T_{\varphi}$ is a Fredholm operator on Hardy space $H^{2}(\partial \mathbb{D})$, and $\operatorname{Ind} T_{\varphi}=-\operatorname{wind} \varphi$. If $\varphi \in C(\overline{\mathbb{D}})$ with $\left|\left(\left.\varphi\right|_{\partial \mathbb{D}}\right)(\zeta)\right|>\epsilon>0$, then $\operatorname{Ind} T_{\varphi}=-$ wind $\left.\varphi\right|_{\partial \mathbb{D}}$ on Bergman space $L_{a}^{2}(\mathbb{D})$. The proof of these index formulas is relative to the topology homotopy of symbol functions, it can not be directly extended to the case of Dirichlet space since we do not know whether there is a $C^{1}$-function $H_{t}$ which is continuous with respect to $t \in[0,1]$ such that $H_{0}=\varphi, H_{1}=\psi$ if $\varphi, \psi \in C^{1}(\overline{\mathbb{D}})$ and wind $\left.\varphi\right|_{\partial \mathbb{D}}=\left.\operatorname{wind} \psi\right|_{\partial \mathbb{D}}$. In this section, we use the 
short exact sequence $(*)$ to prove an analogy of the above index formulas for $C^{1}$-symbols.

Theorem 13. Suppose $\varphi \in C^{1}(\overline{\mathbb{D}})$ such that $T_{\bar{\varphi}}$ is Fredholm on $\mathcal{D}$, then

$$
\operatorname{Ind} T_{\varphi}=-\left.\operatorname{wind} \varphi\right|_{\partial \mathbb{D}} .
$$

Proof. By Theorem 8, if $\varphi, \psi \in C^{1}(\overline{\mathbb{D}})$ such that $T_{\varphi}$ and $T_{\psi}$ are Fredholm on $\mathcal{D}$ and wind $\left.\varphi\right|_{\partial \mathbb{D}}=\left.\operatorname{wind} \psi\right|_{\partial \mathbb{D}}$, then there is a $H_{t} \in C([0,1] \times \partial \mathbb{D})$ such that $H_{t} \in G C(\partial \mathbb{D})$ (the set of invertible elements in $C(\partial \mathbb{D})$ ) for each $t \in[0,1]$ and $H_{0}=\left.\varphi\right|_{\partial \mathbb{D}}, H_{1}=\left.\psi\right|_{\partial \mathbb{D}}$. Note $\xi$ is an isometry isomorphism, so $\xi^{-1}\left(H_{t}\right)$ is continuous with respect to $t$. On the other hand, $H_{t}^{-1}$ is also continuous on $[0,1] \times \partial \mathbb{D}$, and $\xi^{-1}\left(H_{t}\right) \xi^{-1}\left(H_{t}^{-1}\right)=\xi^{-1}\left(H_{t}^{-1}\right) \xi^{-1}\left(H_{t}\right)=\xi^{-1}\left(H_{t} H_{t}^{-1}\right)=$ $[I]$. Hence, $\xi^{-1}\left(H_{t}\right)$ is invertible in $\frac{\mathcal{I}}{\mathcal{K}}$. Furthermore, it is easy to see that $\operatorname{Ind} T_{\varphi}=\operatorname{Ind} T_{\psi}$.

Now suppose $\varphi \in C^{1}(\overline{\mathbb{D}})$ such that $T_{\varphi}$ is Fredholm on $\mathcal{D}$ and wind $\left.\varphi\right|_{\partial \mathbb{D}}=$ $k$, note $T_{z}$ is Fredholm on $\mathcal{D}$ with $\operatorname{Ind} T_{z}=-1=-$ wind $\left.z\right|_{\partial \mathbb{D}}$, let

$$
\tilde{z}^{k}= \begin{cases}z^{k}, & \text { if } k>0, \\ \bar{z}^{-k}, & \text { otherwise }\end{cases}
$$

we see that $\left.\operatorname{wind} \varphi\right|_{\partial \mathbb{D}}=\left.\operatorname{wind} \tilde{z}^{k}\right|_{\partial \mathbb{D}}$, thus $\operatorname{Ind} T_{\varphi}=\operatorname{Ind} T_{\tilde{z}^{k}}$ by above proof, consequently, $\operatorname{Ind} T_{\varphi}=-k=-$ wind $\left.\varphi\right|_{\partial \mathbb{D}}$. The proof is thus complete.

Proposition 14. Suppose $\varphi \in H^{\infty}$ such that $T_{\bar{\varphi}}$ is Fredholm on $\mathcal{D}$, then

$$
\operatorname{Ind} T_{\bar{\varphi}}=-\left.\lim _{r \rightarrow 1^{-}} \operatorname{wind} \bar{\varphi}_{r}\right|_{\partial \mathbb{D}}
$$

where $\varphi_{r}(z)=\varphi(r z)$.

Proof. If $f \in \operatorname{Ker} T_{\bar{\varphi}}$, then for any $g \in \mathbb{D}$,

$$
0=\left\langle T_{\bar{\varphi}} f, g\right\rangle_{\frac{1}{2}}=\langle\bar{\varphi} f, g\rangle_{\frac{1}{2}}=\left\langle\bar{\varphi} f^{\prime}, g^{\prime}\right\rangle=\left\langle\tilde{T}_{\bar{\varphi}} f^{\prime}, g^{\prime}\right\rangle,
$$

since $\left\{g^{\prime} \mid g \in \mathcal{D}\right\}=L_{a}^{2}, \tilde{T}_{\bar{\varphi}} f^{\prime}=0$, hence $\left\{f^{\prime} \mid f \in \operatorname{Ker} T_{\bar{\varphi}}\right\} \subset \operatorname{Ker} \tilde{T}_{\bar{\varphi}}$. Consequently, assume $\tilde{T}_{\bar{\varphi}} f=0, f \in L_{a}^{2}$, set $F(z)=\int_{0}^{z} f d w$, then $F \in \mathcal{D}$, and for any $G \in \mathcal{D}$,

$$
\left\langle T_{\bar{\varphi}} F, G\right\rangle_{\frac{1}{2}}=\left\langle\bar{\varphi} F^{\prime}, G^{\prime}\right\rangle=\left\langle\tilde{T}_{\bar{\varphi}} f, G^{\prime}\right\rangle=0,
$$

so $T_{\bar{\varphi}} F=0$, further $\left\{\int_{0}^{z} f(w) d w \mid f \in \operatorname{Ker} \tilde{T}_{\bar{\varphi}}\right\} \subset \operatorname{Ker} T_{\bar{\varphi}}$. This shows that

$$
\operatorname{dim} \operatorname{Ker} T_{\bar{\varphi}}=\operatorname{dim} \operatorname{Ker} \tilde{T}_{\bar{\varphi}} .
$$

Now assume $f \in \mathcal{D}$ such that $T_{\bar{\varphi}}^{*} f=0$, then for any $g \in \mathcal{D}$,

$$
\begin{aligned}
0 & =\left\langle T_{\bar{\varphi}}^{*} f, g\right\rangle_{\frac{1}{2}}=\langle f, \bar{\varphi} g\rangle_{\frac{1}{2}} \\
& =\left\langle f^{\prime}, \bar{\varphi} g^{\prime}\right\rangle=\left\langle\varphi f^{\prime} g^{\prime}\right\rangle=\left\langle\tilde{T}_{\varphi} f^{\prime}, g^{\prime}\right\rangle,
\end{aligned}
$$


hence $\tilde{T}_{\varphi} f^{\prime}=0$, that is $\left\{f^{\prime} \mid f \in \operatorname{Ker} T_{\bar{\varphi}}^{*}\right\} \subset \operatorname{Ker} \tilde{T}_{\varphi}$.

Similarly to above proof, we have also $\left\{\int_{0}^{z} f(w) d w \mid f \in \operatorname{Ker} \tilde{T}_{\varphi}\right\} \subset \operatorname{Ker} T_{\bar{\varphi}}^{*}$. Hence $\operatorname{dim} \operatorname{Ker} T_{\bar{\varphi}}^{*}=\operatorname{dim} \operatorname{Ker} \tilde{T}_{\varphi}$, consequently,

$$
\operatorname{Ind} T_{\bar{\varphi}}=-\operatorname{Ind} \tilde{T}_{\varphi}=\left.\lim _{r \rightarrow 1^{-}} \operatorname{wind} \varphi_{r}\right|_{\partial \mathbb{D}}=-\left.\lim _{r \rightarrow 1^{-}} \operatorname{wind} \bar{\varphi}_{r}\right|_{\partial \mathbb{D}} .
$$

Acknowledgement. The referee gives many invaluable suggestions and indicates that the functions with derivatives in $H^{\infty}$ are continuous in the closed unit disk. The author is indebted to him for his generous help.

\section{References}

[1] G.F. Cao and Sh.L. Sun, The connectivity of essential spectra of Toeplitz operators with symbols in $H^{\infty}+C$, Chinese Ann. of Math., 1 (1995), 93-102.

[2] R.G. Douglas, Banach algebra techniques in operator theory, Academic Press, 1972.

[3] C.K. Fong, On the essential maximal numerical range, Acta Sci. Math., 41 (1979), 307-315.

[4] McDonald, Fredholm properties of a class of Toeplitz operators on the ball, Indiana Math. J., 26 (1977), 567-576.

[5] R. Rochberg and Z.J. Wu, Toeplitz operators on Dirichlet spaces, Integr. Equat. Oper. Th., 15 (1992), 325-342.

[6] W. Rudin, Function theory in the unit ball of $\mathbb{C}^{n}$, Springer-Verlag, 1980.

[7] Z.J. Wu, Hankel and Toeplitz operators on Dirichlet spaces, Integr. Equat. Oper. Th., 15 (1992), 503-525

Received July 30, 1997 and revised January 15, 1998. This work was supported by National Natural Science Foundation of China.

Sichuan University

Chengdu 610064

CHINA

E-mail address: nic2601@scuu.edu.cu 